\title{
LAS ELECCIONES AL PARLAMENTO GALLEGO
}

En un clima de apatía ciudadana, el día 20 de octubre de 1981, los gallegos acudieron a las urnas para elegir, en el marco de su Estatuto de Autonomía aprobado el 21 de diciembre de 1980, a quienes deberán hacer realidad el autogobierno de una de las tres nacionalidades históricas, si bien se ha dicho que es Galicia la más reacia a ejercer su protagonismo autonómico.

Cuando el 21 de diciembre de 1980 tan sólo uno de cada cuatro gallegos apoyó su Estatuto de Autonomía, un cierto pesimismo se apoderó de los que habían perfilado el proceso de descentralización de España. Es sabido que las tierras gallegas han sido desde las primeras elecciones generales de junio de 1977 un feudo de la abstención. Este importante fenómeno ha sido justificado en incontables ocasiones por lo que se ha venido llamando en medios políticos como «razones técnicas», que no son sino causas político-sociales y obstáculos materiales (la dispersión de las aldeas, el pésimo estado de las comunicaciones, la negligencia de la Administración pública para organizar el voto por correo de los emigrantes...) que harán muy difícil situar la participación gallega ante las urnas en la media nacional.

La abstención gallega se ha mantenido siempre en porcentajes preocupantes: el 38,78 por 100 en junio de 1977 , el 48,58 por 100 en el Referéndum Constitucional, el 48,76 por 100 en marzo de 1979 y el 71,82 por 100 en diciembre de 1980. Es comprensible, pues, que la totalidad de partidos políticos participantes en estos comicios trataran por todos sus medios de borrar el ambiente abstencional del pasado Referéndum. Así, no puede extrañarnos que tanto el objetivo de la campaña institucional (cuyo lema fue «Vota a los tuyos») como el de la carta pastoral de los obispos gallegos y el esfuerzo de todos los dirigentes políticos a nivel regional y nacional, se dirigiera a estimular la participación del electorado gallego en las urnas. El miedo a la abstención eta palpable en todos los medios políticos, sobre todo si se tiene en cuenta que las elecciones al Parlamento gallego han sido consideradas por algunos observadores tanto nacionales como internacionales como un test para la joven democracia española.

En el arranque de la campaña electoral perduraba aún la sensación de desinterés ciudadano que motivó en diciembre pasado la escalofriante cifra de una abstención del 71,82 por 100 del electorado gallego. Alianza Popular ofreció durante esta campaña una imagen muy específica, caracterizada fundamentalmente por la utilización de la lengua gallega y de una abundante simbología: banderas blanquiazules, paisajes típicos y el lema «Galegos coma ti» (Gallegos como tú).

Revista de Derecho Político

Núm. 12. Invierno 1981-1982 
Por su parte, Unión de Centro Democrático utilizó el lema «Defiende lo tuyo» como una apelación directa a los sentimientos supuestamente conservadores del pequeño propietario minifundista.

A su vez, el Partido Socialista Obrero Español de Galicia, a través de su lema «Galicia quiere vivir» y «Haz Galicia viva» trata de presentar una nueva imagen de renovación moderada.

Otras candidaturas que concurrieron a las elecciones al Parlamento gallego fueron las siguientes: el Bloque Nacional Popular Gallego, el Partido Socialista Gallego, el Partido Galleguista, Izquierda Gallega y el Partido Comunista de Galicia.

Una vez finalizada la campaña electoral, los votantes gallegos que acudieron a su cita con las urnas dieron una victoria considerable a Alianza Popular, la cual desbancó a UCD como primer partido de Galicia, a tenor de los resultados definitivos proclamados por la Junta Electoral General. Unicamente el electorado del campo se ha mantenido fiel a los colores centristas, mientras los votantes de las principales ciudades que en las elecciones generales de 1977 y 1979 habían respaldado al partido gubernamental, se han volcado en esta ocasión hacia Alianza Popular.

Los aliancistas vencieron en La Coruña, Santiago, El Ferrol, Lugo, Orense, Pontevedra y Vigo. En todas estas poblaciones el PSOE ocupó el segundo lugar excepto en Pontevedra, donde UCD quedó inmediatamente después de AP con escaso margen de votos.

Las primeras interpretaciones sobre el fracaso de la UCD en Galicia apuntaron como causas fundamentales el voto de castigo urbano hacia la política del Gobierno, así como a la gestión de la Junta de Galicia que ha estado controlada por el partido en el poder.

Por otra parte, el espectacular respaldo del electorado gallego a la derecha no ha impedido que la izquierda y los nacionalistas hayan obtenido más votos en estos comisios que en las municipales de 1979. El PSOE ha subido en número de votos en estas elecciones y el Partido Comunista cosechó malos resultados: tan sólo obtuvo un escaño. Otra fuerza que ha perdido respaldo popular ha sido el Bloque Nacional Popular Gallego. Las restantes organizaciones nacionalistas obtuvieron un reducido apoyo en estas elecciones: el Partido Galleguista no estará en el Parlamento Autónomo y Esquerda Galega ha logrado tan sólo un escaño.

Pilar Mellado Prado 


\section{COMPOSICION DEL PARLAMENTO GALLEGO ${ }^{1}$}

\begin{tabular}{|c|c|c|c|c|c|c|c|}
\hline & A.P. & U.C.D. & P.S.O.E. & P.C.G. & B.N.P.G. & P. Gall. & E. Gall. \\
\hline \multicolumn{8}{|l|}{ La Coruña } \\
\hline $\begin{array}{lll}\text { Votos } & \ldots & \ldots \\
\text { Escaños } & \ldots & \ldots\end{array}$ & $\begin{array}{c}128.901 \\
9\end{array}$ & $\begin{array}{c}76.880 \\
5\end{array}$ & $\begin{array}{c}95.148 \\
6\end{array}$ & $\begin{array}{c}13.519 \\
1\end{array}$ & $\begin{array}{c}26.398 \\
1\end{array}$ & $\stackrel{11.178}{-}$ & $\begin{array}{c}13.176 \\
-\end{array}$ \\
\hline \multicolumn{8}{|l|}{ Lugo } \\
\hline $\begin{array}{lll}\text { Votos } & \ldots & \ldots \\
\text { Escaños } & \ldots & \ldots\end{array}$ & $\frac{43.599}{5}$ & $\begin{array}{c}48.143 \\
6\end{array}$ & $\begin{array}{c}22.431 \\
3\end{array}$ & $\frac{2.046}{-}$ & $\underset{1}{11.182}$ & $\begin{array}{l}5.933 \\
-\end{array}$ & $\underline{1.271}$ \\
\hline \multicolumn{8}{|l|}{ Orense } \\
\hline $\begin{array}{lll}\text { Votos } & \ldots & \ldots \\
\text { Escaños } & \ldots & \ldots\end{array}$ & $\begin{array}{c}40.869 \\
5\end{array}$ & $\begin{array}{c}62.565 \\
7\end{array}$ & $\begin{array}{c}23.612 \\
3\end{array}$ & $\begin{array}{l}2.901 \\
-\end{array}$ & $\begin{array}{l}7.485 \\
-\end{array}$ & $\begin{array}{c}-3.309 \\
-\end{array}$ & $\begin{array}{l}764 \\
-\end{array}$ \\
\hline \multicolumn{8}{|l|}{ Pontevedra } \\
\hline $\begin{array}{lll}\text { Votos } & \ldots & \ldots \\
\text { Escaños } & \ldots & \ldots\end{array}$ & $\begin{array}{l}89.228 \\
7 .\end{array}$ & $\begin{array}{c}87.643 \\
6\end{array}$ & $\begin{array}{c}53.559 \\
4\end{array}$ & $\begin{array}{c}10.572 \\
-\end{array}$ & $\begin{array}{c}17.045 \\
1\end{array}$ & $\underline{12.218}$ & $\begin{array}{c}18.252 \\
1\end{array}$ \\
\hline
\end{tabular}

Indice de participación electoral: 44,99 por 100 .

Indice de abstención: 55,01 por 100 .

Indice de siglas:

U.C.D.: Unión de Centro Democrático.

P.S.O.E.: Partido Socialista Obrero Español.

A.P.: Alianza Popular.

P.C.G.: Partido Comunista de Galicia.

B.N.P.G.: Bloque Nacional Popular Gallego.

P. Gall.: Partido Galeguista.

E. Gall.: Esquerda Galega.

1 Datos suministrados por el Ministerio del Interior.

\section{VOTO POR LOS PRINCIPALES PARTIDOS EN LAS CIUDADES GALLEGAS}

(Elecciones generales de 1979 y regionales de 1981) ${ }^{2}$

\begin{tabular}{|c|c|c|c|c|c|c|}
\hline & \multicolumn{2}{|c|}{$\mathrm{UCD}$} & \multicolumn{2}{|c|}{ PSOE } & \multicolumn{2}{|c|}{ AP } \\
\hline & 1979 & 1981 & 1979 & 1981 & 1979 & 1981 \\
\hline 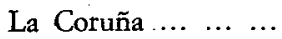 & 38.283 & 10.529 & 21.658 & 28.716 & 16.698 & 33.975 \\
\hline $\begin{array}{llllll}\text { Ferrol } & \ldots & \ldots & \ldots & \ldots\end{array}$ & 13.579 & 3.886 & 8.499 & 10.942 & 5.056 & 11.100 \\
\hline $\begin{array}{ccccc}\text { Santiago } & \ldots & \ldots & \ldots & \ldots\end{array}$ & 14.702 & 3.920 & 4.055 & 6.251 & 5.737 & 13.193 \\
\hline $\begin{array}{lllllll}\text { Lugo } & \ldots & \ldots & \ldots & \ldots & \ldots & \ldots\end{array}$ & 13.185 & 6.233 & 8.149 & 6.982 & 6.784 & 9.405 \\
\hline $\begin{array}{llllll}\text { Orense } & \ldots & \ldots & \ldots & \ldots\end{array}$ & 16.023 & 6.809 & 9.321 & 9.951 & 8.285 & 13.419 \\
\hline $\begin{array}{lllll}\text { Pontevedra } & \ldots & \ldots & \ldots\end{array}$ & 9.936 & 5.778 & 4.586 & 4.979 & 3.578 & 5.796 \\
\hline $\begin{array}{lllllll} & V i g o & \ldots & \ldots & \ldots & \ldots & \ldots\end{array}$ & 36.241 & 13.218 & 19.164 & 18.484 & 17.230 & 30.039 \\
\hline
\end{tabular}

2 FUENTE: Ministerio del Interior. 
EVOLUCION ELECTORAL DE LAS FUERZAS POLITICAS GALLEGAS ${ }^{3}$

(En porcentajes)

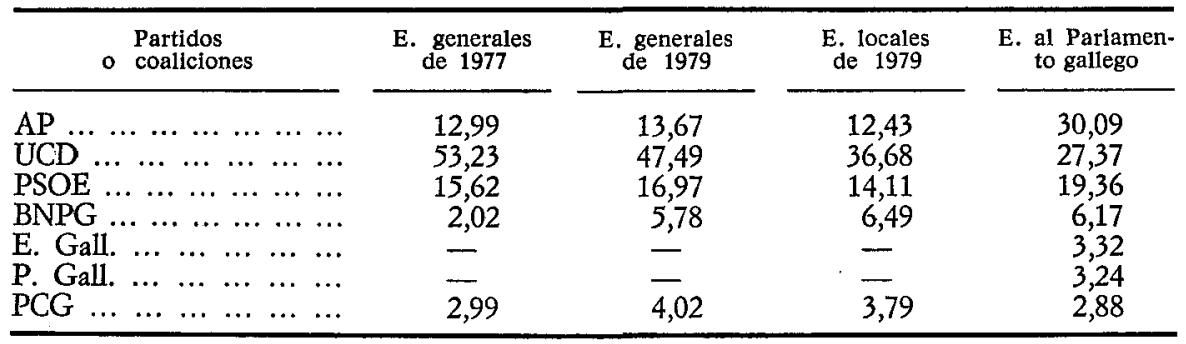

Las siete principales fuerzas políticas gallegas han registrado una evolución desigual desde las elecciones generales del 15 de junio de 1977 hasta los comicios para el Parlamento gallego del pasado 20 de octubre de 1981. Esquerda Galega (E. Gall.) no existía cuando se celebraron las anteriores confrontaciones electorales, y el Partido Galleguista (P. Gall.) no se presentó como tal a las anteriores elecciones sino formando parte de coaliciones. La única coalición presentada el 20 de octubre de 1981 ha sido el Bloque Nacional Popular Gallego (BNPG). La sigia PCG corresponde al Partido Comunista de Galicia.

3 Datos facilitados por el Ministerio del Interior.

\section{PARTICIPACION ELECTORAL EN GALICIA, COMPARATIVAMENTE AL RESTO DE ESPAÑA (en porcentajes)}

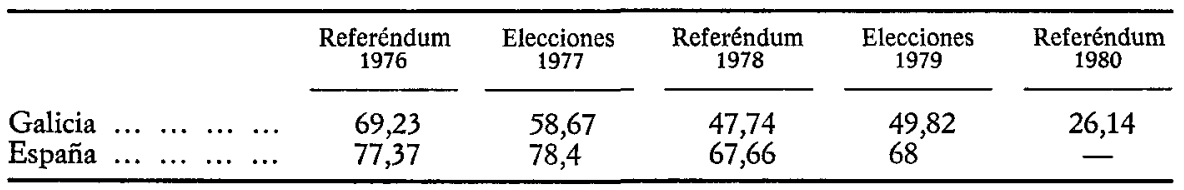

El referéndum de 1976 es el que corresponde a la Ley para la Reforma Política; y el referéndum de 1980 hace referencia al referéndum gallego celebrado el día 21 de diciembre de 1980.

\section{ABSTENCION ELECTORAL EN GALICIA}

(Porcentaje de abstención desde el referéndum para la reforma política hasta las elecciones regionales)

\begin{tabular}{|c|c|c|c|c|c|c|}
\hline Provincia & $\begin{array}{c}\text { Referéndum } \\
1976\end{array}$ & $\begin{array}{c}\text { Elecciones } \\
\text { generales } \\
1977\end{array}$ & $\begin{array}{c}\text { Referéndum } \\
1978\end{array}$ & $\begin{array}{c}\text { Elecciones } \\
\text { generales } \\
1979\end{array}$ & $\begin{array}{c}\text { Elecciones } \\
\text { municipales } \\
1979\end{array}$ & $\begin{array}{c}\text { Elecciones } \\
\text { regionales } \\
1981\end{array}$ \\
\hline 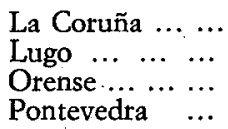 & $\begin{array}{l}29 \\
30 \\
36 \\
28\end{array}$ & $\begin{array}{l}37 \\
43 \\
47 \\
34\end{array}$ & $\begin{array}{l}46 \\
58 \\
61 \\
45\end{array}$ & $\begin{array}{l}47 \\
54 \\
58 \\
45\end{array}$ & $\begin{array}{l}50 \\
43 \\
57 \\
47\end{array}$ & $\begin{array}{l}53 \\
57 \\
58 \\
51\end{array}$ \\
\hline Media regional. & 30 & 39 & 50 & 49 & 49 & 54 \\
\hline
\end{tabular}

FUENTE: Ministerio del Interior. 